\title{
SCIDiC
}

International Journal of Dentistry and Oral Science (IJDOS)

ISSN: $2377-8075$

\section{Implant for Congenitally Missing Maxillary Permanent Cuspid}

Case Report

\author{
Muhamad $\mathrm{AH}^{1 *}$, Watted $\mathrm{N}^{2}$, Proff $\mathrm{P}^{3}$, Borbély $\mathrm{P}^{4}$, Watted $\mathrm{A}^{5}$ \\ ${ }^{1}$ Department of Pediatric Dentistry, University of Athens, Greece. \\ ${ }^{2}$ University Hospital of Würzburg, Clinics and Policlinics for Dental, Oral and Maxillofacial Diseases of the Bavarian Julius-Maximilian-University, \\ Wuerzburg, Germany Triangle R\&D Center, Kafr Qara, Israel and Department of Orthodontics, Arab American University, Jenin, Palestine. \\ ${ }^{3}$ University Hospital of Regensburg, Department of Orthodontics, University of Regensburg, Germany. \\ ${ }^{4}$ Department of Orthodontics, University of Debrecen, Hungary. \\ ${ }^{5}$ Dental school University of Regensburg, Germany.
}

\section{Abstract}

Objective: This case report describes the multidisciplinary approach to treat congenitally missing maxillary canine, how to improve patient's smile using orthodontic fixed appliance, endosseous dental implant, and porcelain veneer to achieve the treatment results of function and esthetic.

Materials and Procedures: Unilateral agenesis of the permanent maxillary canines in healthy individuals is extremely rare. This paper presents the case of a female patient diagnosed with congenital unilateral agenesis of the permanent maxillary canines as well as occlusal abnormalities in the form of left-side crossbite. To restore proper aesthetics and function, interdisciplinary therapeutic treatment was implemented. In the case presented in this paper, the aim of oral rehabilitation was to restore a functional balance by obtaining proper skeletal relationships, create optimal occlusal conditions and obtain arch continuity.

Conclusion: Interdisciplinary treatment combined with orthodontics, implant surgery, and prosthodontics was useful to treat a nonsyndromic oligodontia patient. Especially, the new strategy, implant-anchored orthodontics, can facilitate the treatment more simply with greater predictability.

Keywords: Congenitally Missing Canine; Orthodontic Appliance; Implant Placement; Interdisciplinary Treatment.

\section{Introduction}

Congenital absence of one or more teeth from the dentition has been termed hypodontia. Prevalence of hypodontia in the permanent dentition (excluding 3rd molars) is about $4.5-7.4 \%$ in Caucasians [1] and the most frequently affected teeth are the mandibular second premolar $(3.4 \%)$ and the maxillary lateral incisor $(2.2 \%)$ [2]. Congenital absence of the canines in the permanent dentition is very rare and the reported incidence varies from 0.18 $-0.45 \%[3-5]$. Studies have reported more predilections in females and in the maxilla $[3,4]$.
Dental anomalies associated with congenitally missing permanent canines include agenesis of other teeth, microdontia, malocclusion, and retained primary teeth [4]. Congenital agenesis of permanent canines in both maxilla and mandible is extremely rare [6].

The etiology of such an anomaly is obscure. Whilst racial difference in prevalence suggests that genetic factors may be a more probable reason to the congenital absence of teeth, variable etiology exists including hereditary, environmental or endocrine disturbances [6]. Recent studies have reported that synergistic interaction of genes Pax9 and Msx1 may cause human tooth agenesis $[7,8]$.

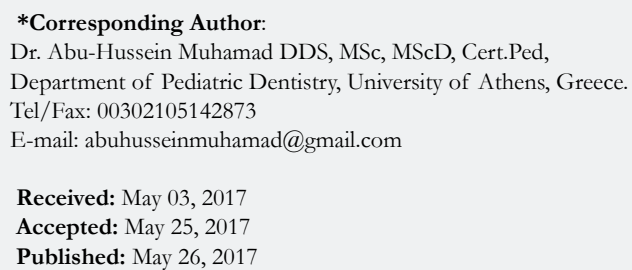

Copyright: Muhamad $\mathbf{A H}^{\circ}$ 2017. This is an open-access article distributed under the terms of the Creative Commons Attribution License, which permits unrestricted use, distribution and reproduction in any medium, provided the original author and source are credited. 
Agenesis of permanent teeth has been strongly correlated to the absence of corresponding primary predecessors $[6,8,9]$. The present case showed agenesis of bilateral maxillary permanent canines, mandibular right permanent canine, and both the mandibular central incisors, while the corresponding predecessors were clinically present [8].

Treatment approach has to be case specific and depends on condition of primary predecessor, number of missing teeth, status of occlusion/occlusal condition and patient/parent's preferences $[10,11]$. Timely extraction of primary predecessors can be considered to allow spontaneous space closure with or without further orthodontic alignment or the primary canines can be retained until replacement with a suitable restoration when they are lost [12-14]. Our case displayed little or no resorption of retained primary canines at the time of diagnosis.

Primary canines without permanent successors might have reasonably longer survival rates and therefore would be functionally advantageous in subjects affected with severe hypodontia [14-16]. Retaining the primary teeth in such cases may impede alveolar resorption until adolescent stage and help to preserve the volume of bone, for replacement with end-osseous implants in favorable locations, at a later time, without the need for bone grafting [1719].

Minimally invasive dentistry can be defined of maximal preservation of healthy dental structures. It would be inappropriate to remove enamel and dentin excessively because it will reduce ability to bond restorative material $[16,17]$. Especially, patients with dental agenesis, it would be the best way to replace space with endosseous dental implant instead of doing fixed partial denture which is reducing enamel and dentine tooth structure of the abutments. Subgingival margins are required in esthetic situations, but these are associated with increased gingival inflammation $[6,11,14,15]$. While some clinicians may suggest that a resin-bonded prosthesis is a viable option, clinical experience has shown that these resinbonded pontics do not have long-term success rate if the teeth are not prepared aggressively enough for mechanical retention $[12,14]$. In these cases, implants were represented the most conservative treatment to rehabilitate patients with absence of permanent teeth. For the dental implant, edentulous area should be evaluated for example quality, quantity of bone and amount of space to confirm the possibility of surgical implant installation without compromising the esthetic result [15-17]. The appropriate amount of space for implant and crown is determined by size of implant, surrounding living tissue, crown shape, occlusion and the recurring esthetic dental (RED) proportion from patient smiling $[6,10,13,15]$. As the result, an interdisciplinary approach including orthodontics, periodontics, surgery implant placement and operative treatment is taken into account $[6,10,13,17]$.

The aim of this paper is to present the interdisciplinary treatment of hypodontia of permanent canine in an adult female.

\section{Case Report}

A female patient, aged 21, visited an orthodontist to embark on orthodontic treatment. The patient still had left primary canines, but otherwise he was generally healthy and had not suffered any other health problems. The patient had no history of having suffered any trauma within the facial skeleton. In addition, he was not aware of any instances of congenitally missing teeth in his family.

A clinical examination revealed a slight asymmetry of facial features between the left and right sides of the face, retracted subnasal region, and a posterior position of the upper lip position relative to Ricketts' e-line. Intraoral examination revealed the presence of persistent primary canine teeth: 63 (Class II mobility); as well as the presence of permanent teeth in the maxilla and mandible except maxillary canines and third molars. In addition, complete leftside cross-bite was diagnosed involving teeth 22, 23, $24,25,26$ and 27 as well as a deviated midline between the maxilla and the mandible.

The presence of persistent deciduous teeth 63 with some root resorption visible; hypodontia of permanent teeth 23 ; and the presence of the remaining permanent teeth except the third molars.

A treatment plan which involved orthodontic as well as implantprosthetic procedures was prepared and presented to the patient, who accepted it and gave his consent to initiating the treatment.

Orthodontic treatment with fixed appliances on the upper and lower dental arches was intended to widen the jaw, eliminate cross-bite on the left side, restore correct lateral relationships and reduce overbite. The treatment assumed leaving the persistent primary teeth in place as long as possible in order to preserve the volume of the alveolar bone with a view to a planned future implantation after the extraction of the left primary maxillary $\mathrm{Ca}$ nine. The treatment with fixed appliances lasted 18 months, and the desired therapeutic effects were achieved (Figure. 1a-d).

Figure 1a-d.

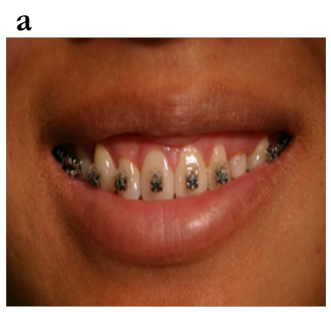

b

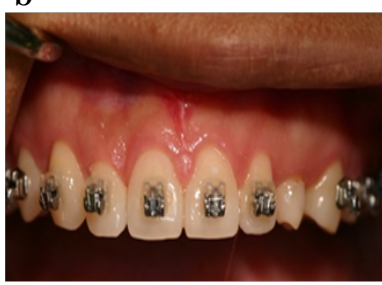

c

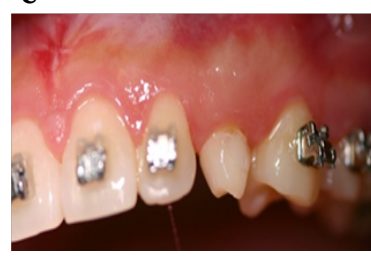

d

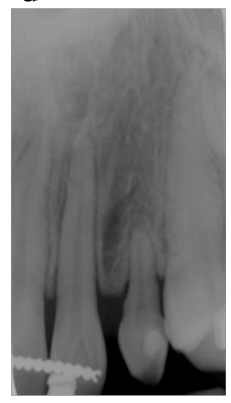


Upon the completion of orthodontic treatment, primary teeth 63 were extracted. An analysis of the alveolar ridge performed using cone beam computed tomography showed a sufficient volume of bone for the placement of implants. Implantation was performed under local anesthesia. First, the mucoperiosteal flap was elevated and the implant sites were prepared, into which $11 \mathrm{~mm} \times 3.5 \mathrm{~mm}$ Astra implants were inserted (Figure. 2-8 ).

The wound was sutured and Solcoseryl was applied. The healing process proceeded without any complications. After a week, the sutures were removed. Four months later, the implants were uncovered and healing abutments were attached to the implant fixtures (Figure. 9).

Approximately three weeks after the uncovering of the implants, under infiltration anesthesia, retraction cords were placed and the upper incisors were prepared for porcelain veneers. An impression was taken in a one-step technique. Temporary veneers were then attached to the prepared teeth. After a week the final restorations were fixed using Variolink Veneer . Next, transfer devices were used to take an impression for zirconia-based crowns. Afterwards zirconia connectors were fitted and the finished crowns were seated. Prior to all the prosthetic procedures (Figure. 10, 11, 12). Figure.13.

Figures 2-9.

Figure 2.

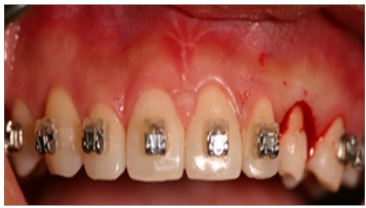

Figure 4.

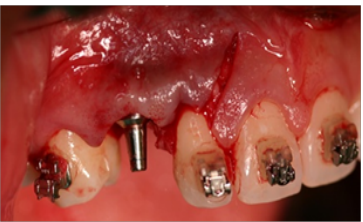

Figure 6.

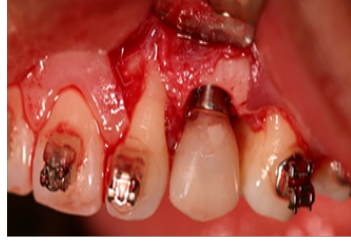

Figure 8.

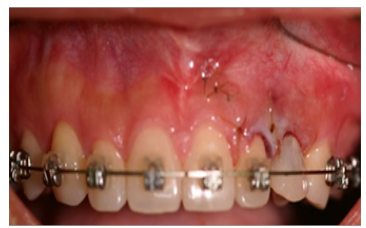

Figure 3.

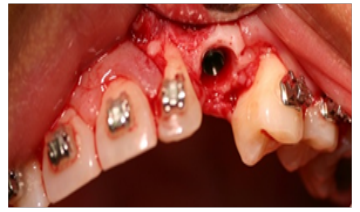

Figure 5.

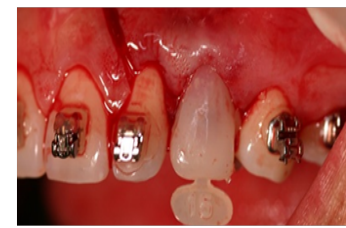

Figure 7.

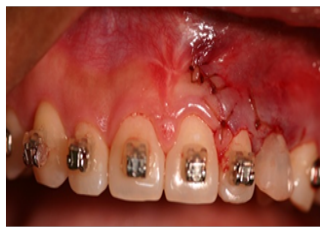

Figure 9.

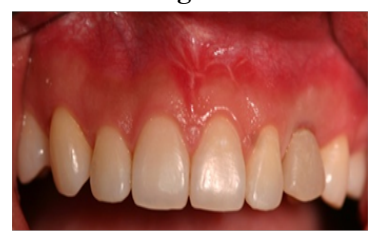

Figures 10-13.

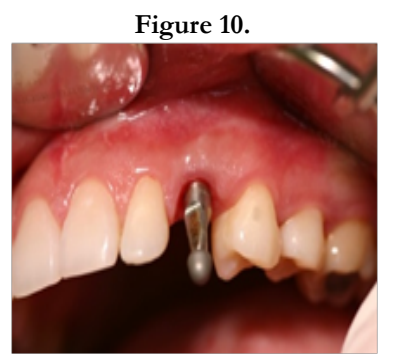

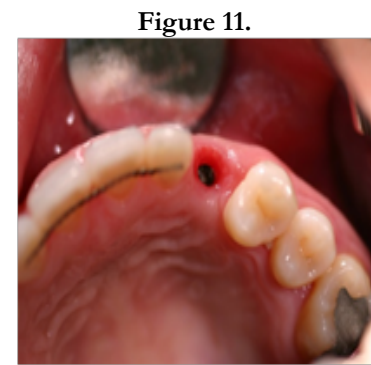

Figure 13.

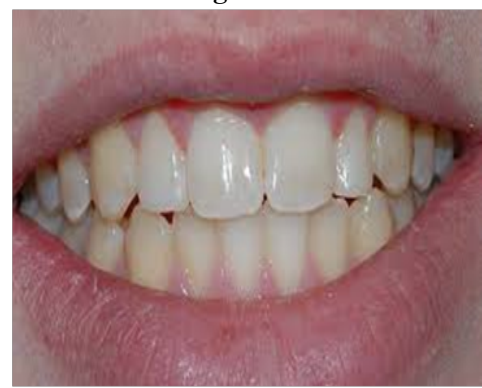

Figure 12.

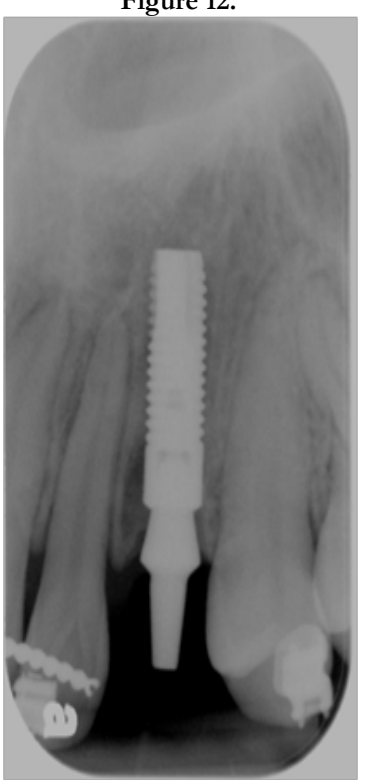




\section{Discussion}

A congenitally absent tooth for maxillary permanent canines is rare. There are several treatment options for replacing the congenitally missing maxillary canine. These include removable denture prostheses, conventional fixed partial denture, orthodontic repositioning of premolars to close the edentulous spaces, and single-tooth implant $[11,12,16]$. In this case patient could be treated in another way by extraction teeth. The extraction teeth would be teeth 63 . The first maxillary premolars will be used to substitute for the canines. Figure 13. The positive side to this treatment method is that the patient will not require to do the dental implant restoration. This treatment prepared spaces for placing implants which in retrospect provided many advantages.

The treatment time is less than in the case of the extraction method. It don't have to remove healthy teeth from patient which he doesn't need. The most importantly the patient's facial profile will remain as it is [17, 20-22].

The use of intraosseous implants for the reconstruction of partial or complete tooth loss is a well-documented method of treatment, and the phenomenon of their osseointegration has been extensively explored. Increasing pressure from patients in terms of the aesthetic appearance of dental restorations forces dental practitioners to choose the best type of restoration as well as continuously modifying surgical treatment protocols [18-20, 22].

The absence of even a single tooth which has not been prosthetically restored results in irreversible adverse changes to the surrounding hard and soft tissues [23]. The easiest method of replacing a missing tooth is a fixed prosthetic restoration consisting of splinted crowns supported by the neighboring teeth. However, such a solution is invasive and destructive to the pulp and hard tissues of the teeth adjacent to the gap. In addition, it also does not prevent the loss of alveolar bone [24, 25].

An alternative solution may be the use of restorations based on intraosseous implants. An essential prerequisite for effective and aesthetic implantation, though, is the availability of an appropriate amount of surrounding and supporting tissue [11, 12, 14, 26, 27].

A sufficient amount of bone at the implant site, as was the case in the patient described here, usually guarantees a successful outcome. However, in situations where the volume of bone is insufficient and when additional periodontal problems occur, it is advisable to perform tissue augmentation in the form of bone grafts, connective tissue grafts or guided bone regeneration [13, 15-17].

Another important factor which affects the long-term clinical results of implant treatment is the geometry of the implant surface. Implants with a smooth machined surface show the greatest rate of failure, reaching $18.5-26 \%$ for $7 \mathrm{~mm}$ implant lengths. Increasing the surface geometry of the implant through appropriate conditioning which produces a more porous structure, such as plasma spraying or the use of special granules, increases contact with the bone tissue. This means that the forces are distributed over a larger surface of the implant $[26,27]$.
Three situations are possible depending on the available space in the arch and its manipulation [28];

(a) - Orthodontic treatment can compensate for the absence of a canine by moving the first premolar into its site as a component of the over-all correction of the malocclusion dysmorphism.

(b) - If the clinical situation allows, that is if adequate space is available and no crowding exists elsewhere in the dental arches, the treating dentist can replace the missing canine prosthetically without prior orthodontic treatment.

(c) - Orthodontic treatment can create the coronal and apical space necessary for insertion of an implant or fixed or removable dental prosthesis as a single procedure or in the course of correcting other aspects of malocclusion, if they are present.

Replacements without any preliminary orthodontic treatment when they deem patients have reached the appropriate age, which is at the end of the facial growth period [29]. Until this time, they may decide to use temporary cemented bridges, bonded artificial teeth, or removable plates bearing an acrylic tooth as space maintainers and to improve the patients' appearance. Patients and families must be made fully aware of the possibility that a single implant placed in a young patient may not accommodate itself to alveolar development throughout life thus falling out of occlusion and acquiring an unsightly gingival festooning not in harmony with those of adjacent teeth [11, 12, 14, 16-18].

A third solution requiring a preliminary orthodontic stage to prepare for a satisfactory prosthetic replacement must be envisaged, one that would be accompanied by all the advantages and, primarily, disadvantages elucidated for immediate placement of a prosthesis or an implant $[30,31]$.

This case report presents the clinical manifestations of prolonged retention of the primary maxillary canines due to congenitally missing of permanent canine. Improper spaces of the maxillary canines for placing implants was corrected by orthodontic treatment. Abnormal tooth size and shape restored by ceramic veneers at incisor teeth. As the result of treatment, excellent function and esthetic of patient was obtained with minimal invasiveness, while the patient regained confidence because of his new, esthetically pleasing smile.

\section{Conclusion}

Interdisciplinary treatment combined with orthodontics, implant surgery, and prosthodontics was useful to treat a nonsyndromic oligodontia patient. Especially, the new strategy, implant anchored orthodontics, can facilitate the treatment more simply with greater predictability.

\section{References}

[1]. Lombardo C, Barbato E, Leonardi R (2007) Bilateral maxillary canines agenesis: a case report and a literature review. Eur J Paediatr Dent. 8(1): 38-41.

[2]. Nestorowicz-Obrzut K, Jarka J (2013) Bilateral agenesis of permanent maxillary canines - two case reports. Forum Ortod. 9(2): 124-139.

[3]. Muhamad Abu-Hussein, Nezar Watted, Abdulgani Azzaldeen, Mohammad Yehia, Obaida Awadi, et al., (2015) Prevalence of Missing Lateral Incisor Agenesis in an Orthodontic Arabs Population in Israel (Arab48). Int J Public Health Res. 3(3): 101-107.

[4]. Muhamad Abu-Hussein, Nezar Watted, Ali Watted, Yosef Abu-Hussein, 
Mohammad Yehia, et al., (2015) Prevalence of Tooth Agenesis in Orthodontic Patients at Arab Population in Israel. Int J Public Health Res. 3(3): 77-82.

[5]. Cho S, Lee C, Chan J (2004) Congenitally missing maxillary permanent canines: report of 32 cases from an ethnic Chinese population. Int J Paediatr Dent. 14(6): 446-450.

[6]. Abu-Hussein M, Abdulgani A, Watted N, Zahalka M (2015) Congenitally Missing Lateral Incisor with Orthodontics, Bone Grafting and Single-Tooth Implant: A Case Report. J Dent Med Sci. 14(4): 124-130.

[7]. Guna Shekhar M, Srinivas Rao K, Dutta B (2011) A rare case of congenital absence of permanent canines associated with other dental anomalies. J Clin Exp. Dent. 3(1): e70-72.

[8]. Abu-Hussein M, Watted N, Yehia M, Proff P, Iraqi F (2015) Clinical Genetic Basis of Tooth Agenesis. J Dent Med Sci. 14(12): 68-77 DOI: 10.9790/0853-141236877

[9]. Koc N, Cagirankaya LB, Akkaya N (2014) Unilateral maxillary canine agenesis: a case report and literature review. Case Rep Dent. 2014: 685014. doi: $10.1155 / 2014 / 685014$

[10]. Abdulgani A, Kontoes N, Chlorokostas G, Abu-Hussein M (2015) Interdisciplinary Management Of Maxillary Lateral Incisors Agenesis With Mini Implant Prostheses: A Case Report. IOSR-JDMS. 14(12): 36-42.

[11]. Abu-Hussein Muhamad Chlorokostas Georges, Abusalih Ahmet, Ismail Hakki Bayraktar, Abdulgani Azzaldeen (2016) Immediate Implant Placement and Loading in Esthetic Zone. IOSR-JDMS. 15(1)0: 71-79. DOI: 10.9790/0853-15187179

[12]. Nezar Watted, Muhamad Abu-Hussein (2016) Multidisciplinary Aesthetic Dental Treatment; Peg lateral with Congenitally Maxillary lateral Incisors. IOSR-JDMS. 15(10): 83-91. DOI: 10.9790/0853-1510018391

[13]. Muhamad AH, Azzaldeen A, Nezar W, Mohammed Z (2015) Esthetic Evaluation of Implants Placed after Orthodontic Treatment in Patients with Congenitally Missing Lateral Incisors. J Adv Med Dent Scie Res. 3(3): 110118.

[14]. Abusalih A, Ismail H, Abdulgani A, Chlorokostas G, AbuHussein M (2015) Interdisciplinary Management of Congenitally Agenesis Maxillary Lateral Incisors: Orthodontic/Prosthodontic Perspectives. IOSR-JDMS. 15( 1): 90-99. DOI: 10.9790/0853-15189099

[15]. Abu-Hussein M, Chlorokostas G, Watted N , Abdulgani A , Jabareen A (2016) PreProsthetic Orthodontic Implant for Management of Congenitally Unerupted Lateral Incisors - A Case Report. ISOR-JDMS. 15: 99-104.

[16]. Abu-Hussein M, Watted N, Abdulgani A (2016) Managing congenitally missing lateral incisors with single tooth implants. Dent Oral Craniofac Res. 2(4): 318-324.

[17]. Mai A, Azzaldeen A, Nezar W, Chlorokostas G, Muhamad AH (2016) Extraction and Immediate Implant Placement with Single-Stage Surgical Pro- cedure: Technical Notes and a Case Report. J Dent Med Sci. 15: 95-101.

[18]. Abu-Hussein M, Georges C, Watted N, Azzaldeen A (2016) A Clinical Study Resonance Frequency Analysis of Stability during the Healing Period. Int J Oral Craniofac Sci. 2(1): 065-071. DOI: 10.17352/2455-4634.000021

[19]. Abu-Hussein M, Watted N, Shamir D (2016) A Retrospective Study of the AL Technology Implant System used for Single-Tooth Replacement. Int J Oral Craniofac Sci. 2(1): 039-046. DOI: 10.17352/2455-4634.000017

[20]. Fradeani M, Barducci G (2004) Tooth analysis. Esthetic rehabilitation in fixed prosthodontics. (1st Edn), Hanover Park, IL: Quintessence Publishing: USA. 137-241.

[21]. Adilson YF, Eduardo JF, Jose M (2008) Esthetic and functional restoration for an anterior open occlusal relationship with multiple diastemata: A multidisciplinary approach. J Prosthet Dent. 99(2): 91-94.

[22]. Arthur MR, Stephen F Rosenstiel (2012) Esthetic considerations related to bone and soft tissue maintenance and development around dental implants: Report of the Committee on Research in Fixed Prosthodontics of the American Academy of Fixed Prosthodontics. J Prosthet Dent. 108(4): 259-267.

[23]. Abdulgani Azzaldeen, Nezar Watted, Abdulgani Mai, Péter Borbély, Muhamad Abu-Hussein (2017) Tooth Agenesis; Aetiological Factors. ISORJDMS. 16(1): 75-85. DOI: 10.9790/0853-1601057585

[24]. Funato A, Ishikawa T (2011) 4D Implant Therapy: Esthetic Considerations for Soft Tissue Management. 2011: 9-15.

[25]. Garber DA, Salama MA, Salama H (2001) Immediate total tooth replacement. Compend Contin Educ Dent. 22(3): 210-8.

[26]. Bajali M, Abdulgani Azz, Abu-Hussein M (2014) Extraction and immediate implant placement, and provisionalization with two years follow-up: a case report. Int J Dent Health Sci. 1(2): 229236.

[27]. Muhamad AH, Azaldeen A, Watad M, Watted N (2014) Tooth extraction, immediate implant placement: a case report. Asian Pac J Health Sci. 1(4): 543-549.

[28]. Abu-Hussein Muhamad, Watted Nezar, Abdulgani Azzaldeen (2015) The Curve of Dental Arch in Normal Occlusion. Open Sci J Clin Med. 3(2): 47-54.

[29]. Stefanos K, Christina P, Panagiotis A, Asterios D (2007) Provisional Restorations for Optimizing Esthetics in Anterior Maxillary Implants: A Case Report. J Esthet Restor Dent. 19(1): 6-18.

[30]. Adilson YF, Eduardo JF, Jose M (2008) Esthetic and functional restoration for an anterior open occlusal relationship with multiple diastemata: A multidisciplinary approach. J Prosthet Dent. 99(2): 91-94.

[31]. Jonathan DL (2012) Aesthetic Crown Lengthening: A Step by Step Surgical Guide and Biologic Considerations. Alpha Omegan. 102(4): 133-141. 